\title{
Qualitative study of the quality of sleep in marginalized individuals living with HIV
}

\author{
This article was published in the following Dove Press journal: \\ Patient Preference and Adherence \\ 13 June 2013 \\ Number of times this article has been viewed
}

\author{
Parya Saberi' \\ Megan Comfort ${ }^{2}$ \\ Nicolas Sheon' \\ Mallory O Johnson' \\ 'Department of Medicine, University \\ of California, San Francisco, CA, USA; \\ ${ }^{2}$ RTI International, San Francisco, \\ CA, USA
}

\begin{abstract}
Sleep disturbances have been reported to be higher in human immunodeficiency virus (HIV)-infected individuals compared to the general population. Despite the consequences of poor quality of sleep (QOS), research regarding sleep disturbances in HIV infection is lacking and many questions regarding correlates of poor QOS, especially in marginalized populations, remain unanswered. We conducted one-on-one qualitative interviews with 14 marginalized HIV-infected individuals who reported poor QOS to examine self-reported correlates of sleep quality and explore the relationship between QOS and antiretroviral adherence. Findings suggest a complex and multidimensional impact of mental health issues, structural factors, and physical conditions on QOS of these individuals. Those reporting poor QOS as a barrier to antiretroviral adherence reported lower adherence due to falling asleep or feeling too tired to take medications in comparison to those who did not express this adherence barrier. These interviews underscore the importance of inquiries into a patient's QOS as an opportunity to discuss topics such as adherence, depression, suicidal ideation, and substance use.
\end{abstract}

Keywords: adherence, HIV/AIDS, interviews, qualitative research, sleep

\section{Introduction}

Sleep disturbances have been reported in many studies since early in the human immunodeficiency virus (HIV) epidemic and have been deemed to be higher in HIV-infected individuals in comparison to the HIV-seronegative population. ${ }^{1-4}$ Nearly $73 \%$ of people living with HIV report sleep disturbances, ${ }^{5}$ as compared to $10 \%-35 \%$ of the general population. ${ }^{6,7}$ Currently, over 30 years into the HIV epidemic, the exact mechanisms of these sleep difficulties are still unknown and understudied, but are hypothesized to be correlated with viremia, psychiatric illnesses, illicit drug or alcohol use, or use of antiretroviral (ARV) medications. ${ }^{5,8-15}$

Decreased quality of sleep (QOS) may directly or indirectly, through its association with depressive symptoms, result in nonadherence. ${ }^{12,16,17}$ The personal and public health significance of adherence is evident in that small reductions in ARV adherence have been associated with loss of virologic control and treatment failure, emergence and transmission of drug-resistant virus, and disease progression. ${ }^{18-23}$ Understanding the reasons behind sleep disorders and the impact of poor sleep quality on daily life is essential because sleep disturbances have been associated with impaired memory, attention, and concentration; ${ }^{24,25}$ increased psychiatric disorders, ${ }^{26}$ and higher health care utilization. ${ }^{27}$ In addition, sleep disorders possess an inflammatory component, which is thought to be the basis for increased risk of coronary artery disease and increased cardiovascular morbidity and mortality. ${ }^{8-10,28-33}$ In brief, the recognition
Correspondence: Parya Saberi

University of California, Department

of Medicine, UCSF Box 0886,

San Francisco, CA, 94105, USA

Tel +l 4I55978|44

Email parya.saberi@ucsf.edu 
and timely treatment of poor QOS may result in improved ARV adherence and reduced cardiovascular disease, and therefore is an important part of the clinical management of HIV-infected individuals.

The interaction of ARV nonadherence and hypothesized correlates of sleep difficulties, such as psychiatric disorders and drug and alcohol use, is particularly pronounced in the marginalized HIV-infected population; however, research regarding sleep disturbances in this population is lacking. Therefore, we conducted a qualitative study to deepen our understanding of poor QOS in relation to mental and physical health aspects of the lives of marginalized HIV-infected individuals and to explore ARV adherence in the context of poor QOS. In this paper, we report our findings regarding self-reported correlates of poor QOS, the relationship between QOS and ARV adherence, and other themes that emerged through data analysis.

\section{Methods}

\section{Setting}

We conducted one-on-one, semistructured, qualitative interviews that were nested in the Preparing patients to start Antiretroviral Therapy project (PATH, R01MH079700), a randomized controlled trial evaluating an intervention to enhance information, motivation, and behavioral skills of marginalized $\mathrm{HIV}$-infected adults with a $\mathrm{CD}_{4}^{+}$cell count $<500$ cells $/ \mathrm{mm}^{3}$ in order to increase the rate of ARV uptake. The PATH Project targeted members of the population for whom ARVs were strongly advised and who were most likely to die from AIDS-related illness or were at increased risk of transmitting HIV to others if not engaged in effective treatment. Throughout the course of this trial, participants' ARV initiation and adherence was monitored. Participants in the PATH project were $\geq 18$ years of age, had a confirmed HIV-positive test, reported knowing their HIV serostatus for at least 6 months, had not taken any ARV medications for the prior 30 days, had never taken a continuous course of ARVs for more than 30 days, had a confirmed $\mathrm{CD}_{4}^{+}$cell count $<500$ cells $/ \mathrm{mm}^{3}$, were able to provide informed consent for research, and spoke English. Individuals with cognitive impairment, active psychosis, or significant confusion were excluded from the project.

\section{Participants}

Participants in the qualitative substudy were 14 HIV-infected adults from the waitlist control arm of the PATH Project who reported poor QOS, as defined by two questions in the Pittsburg Sleep Quality Index: ${ }^{34}$ (1) "During the past month, how many hours of actual sleep did you get at night? (This may be different than the number of hours you spent in bed)," for which participants could enter the number of hours slept between 0-24 hours; and (2) "During the past month, how would you rate your sleep quality overall?", where responses included (0) very good, (1) fairly good, (2) fairly bad, and (3) very bad. We purposefully sampled those who reported 6 or fewer hours of sleep at night or those reporting "fairly bad" or "very bad" sleep quality in the past month. During the follow-up period of the PATH Project, several participants in the control arm initiated ARVs; therefore, we were able to sample among those who had initiated ARVs in order to examine the relationship between QOS and ARV adherence, as well as those who had not initiated ARVs. Exclusion criteria consisted of cognitive impairment or inability to participate due to intoxication with alcohol or use of other illicit substances at the time of the interview. Participants who met the inclusion criteria were contacted by the research staff, were consecutively enrolled on a first-come-first-serve basis, and received $\$ 40$ in the form of gift vouchers for participation. The University of California, San Francisco Committee on Human Research granted approval of this project, and participants signed an informed consent form prior to initiation of the interview.

\section{Data collection}

Data were collected through semistructured, one-time, 1.5- to 2-hour interviews, during which we focused on narratives of the participants' experiences with poor QOS. All interviews were conducted in a private room at the Tenderloin Clinical Research Center in San Francisco, California. The first author conducted all interviews from February through October of 2010. The interview guide, which was specifically created for this study, consisted of four key sections: (1) demographics and social history, consisting of questions about the participant's daily schedule, living and family situation, as well as disclosure of HIV status; (2) HIV treatment history (as applicable), including questions regarding participant's ARV medications, ARV adverse effects, number of doses missed in the past week, and reasons for missed doses; (3) sleep experience, including questions regarding timeline of sleep problems, self-perceived causes of sleep problems, impact of sleep difficulties on daily life, methods of dealing with sleep problems, experience of dreams, perceived effect of ARVs on QOS, and impact of sleep difficulties on ARV adherence; and (4) additional questions regarding depression, substance use, incarceration, relationship with health care providers, and support from family and friends. ARV adher- 
ence was calculated by dividing the total number of doses taken per week by the number of expected doses based on participants' ARV regimen. Throughout the process of data collection and concurrent analysis, we made minor changes to the interview guide in order to probe more deeply into topics that our initial analysis indicated were important with regard to QOS.

\section{Analysis}

We employed an inductive approach to gain a deeper understanding of participants' experiences with and perspectives on factors associated with poor QOS and the impact of sleep quality on ARV adherence. This approach entails "detailed readings of raw data to derive concepts, themes, or a model." 35 Immediately following each interview, the first author wrote memos capturing salient points, initial thoughts, and impressions of the interview. Additionally, for all interviews, the first author summarized the participant's daily schedule, definition of a good night's sleep, type(s) of sleep disturbance, perceived causes of sleep difficulties, methods that the participant has tried to deal with poor QOS, experiences of dreams, ARV adherence, mental health issues, and physical conditions. These analytical memos and interview summaries were shared with the second author, who listened to the interviews and provided a secondary perspective and rendered new or alternative observations. Next, the digital audio recordings of the interviews were transcribed verbatim and entered into the qualitative data analysis software, Transana (Wisconsin Center for Education Research, Madison, WI, USA). This software allowed us to segment, code, and structure our data for analysis. We used an inductive coding process in which the first author read and analyzed transcripts, field notes, and memos line by line; organized interview contents; developed a set of codes based on a priori and emerging themes; and subsequently clustered these codes into categories. ${ }^{36}$ Finally, the first, second, and third authors worked together to condense, develop, and refine the codes and categories and to organize the data within this analytical scheme.

\section{Results}

We interviewed 14 HIV-infected individuals with a mean age of 44 years who were $79 \%$ male at birth, $43 \%$ Black, and $36 \%$ White. At the PATH Project baseline, mean HIV viral load was $4.46 \log _{10}$ copies/mL and mean $\mathrm{CD}_{4}^{+}$cell count was 222 cells $/ \mathrm{mm}^{3}$. All participants reported having been homeless, 79\% reported having been incarcerated, and $86 \%$ had a high school diploma or greater. Mean number of hours of actual sleep per night during the past month was 4.6 hours (standard deviation $=1.7$ ), and the majority of participants rated their overall QOS in the past month as "fairly bad."

When asked to define good sleep quality, participants' responses included uninterrupted sleep for 8 hours, feeling rested and energized upon awakening, feeling relaxed and without fear or worry during the night, and not experiencing repeated awakenings due to physical issues such as muscle spasms or diarrhea. Many participants reported not having given much thought to the root of their sleep problems, not having fully considered the potential reasons for their sleep difficulty, and infrequently discussing these issues with their health care providers. Many stated that their reason for rarely discussing their sleep with their physician was the presence of other pressing health issues (such as cancer, trauma, or homelessness), the need to stay alert in the street (if homeless) and therefore not wanting to fall into deep sleep, the presumed quick-to-prescribe nature of this interaction, and the participants' aversion to taking even more medications.

All participants reported consequences of their poor QOS, such as difficulty concentrating, frequently falling asleep during the day, memory loss, constant fatigue, and needing to change their daily routine due to exhaustion. Participants reported experimenting with a variety of techniques to help improve their sleep. These methods included the use of a variety of substances, including alcohol, cigarettes, marijuana, other street drugs (such as crack and cocaine), over-the-counter medications (such as diphenhydramine), and prescription medications (such as zolpidem, trazodone, hydrocodone, mirtazapine, quetiapine, and clonazepam), as well as nonpharmacologic methods such as sleep deprivation, meditation, reading, creative writing, watching television, exercising and exertion, and sleeping in a safe indoor space, such as a hospital emergency room.

We discuss salient themes that emerged through our data analysis. While acknowledging considerable overlap and interplay among them, we group these themes into the following categories: ARV adherence, mental health issues, structural factors, and physical conditions.

\section{Sleep and ARV adherence}

Since enrollment in the PATH Project, 71\% $(\mathrm{n}=10)$ of participants had initiated ARVs at the time of their respective interviews; $50 \%$ were taking a protease inhibitor-based regimen, 14\% had started an efavirenz-based regimen, and $7 \%$ were on an integrase inhibitor-based 
regimen. Among these ten participants, self-reported ARV adherence in the previous week was approximately $95 \%$ (range $=73 \%-100 \%)$.

The topic of ARV nonadherence due to falling asleep, being too tired to take medications, or lack of concentration due to fatigue was brought up by participants in five interviews. Adherence among these five individuals was $90 \%$ (range $=73 \%-97 \%$ ) and falling asleep or lack of sleep accounted for approximately one to four missed doses per week, which represented $50 \%-100 \%$ of their total weekly missed doses. These doses were predominantly in the evening.

Participant: I get tired real easy a lot. I don't sleep that much even though I'm always tired.

Interviewer: For the past week when you [said that you] missed three or four doses, how many of that do you think would have been somehow related to your sleep?

Participant: More than half; at least more than half. [31-year-old Latino male on ARVs]

\section{Sleep and mental health issues}

Among the participants, the mean Center for Epidemiologic Studies Depression Scale score was 33 (indicative of major depression). All participants stated that they felt depressed or had been diagnosed with depression. All had been prescribed antidepressant medications and referred for mental health services during the course of their clinical care, but most of those adhering to their antidepressant medications and receiving mental health services indicated that their QOS had not significantly improved. Depression influenced participants' QOS by impacting their ability to fall asleep and stay asleep, experience of dreams, initiative to get out of bed in the morning, and need to take frequent naps. Participants directly attributed their poor QOS to struggling with depression, as articulated by a 45-year-old Black male on ARVs:

$[\ldots]$ in my depression I think what keeps me up a lot is that it has a tendency to make my mind wonder "what if" [...] I can lay in the bed and not go to sleep, I just don't want to get up $[\ldots]$ sometimes I've laid in the bed on a weekend just two or three days and just lay there and not sleep. I'm just there [...] I don't want to do anything [...] I don't want to cry, I don't want to care [...] I get to the point where I don't think it's just worth anything.

Although most participants did not attribute changes in their sleep quality to HIV seroconversion, many discussed the mental stress and worry after testing positive, the anxiety of living with HIV, and the fear of infecting others as severely impacting their QOS. As expressed by a 55-year-old Black female not on ARVs:

Fear of dying and having HIV, just a fear [of] having HIV [...] you need to think about being ashamed. And, sleeping, it's got a lot to do with your resting inside, mentally. If you can't rest inside mentally, you're not sleeping nowhere, no matter where you go.

Or in the words of a 35-year-old White male on ARVs:

When I'm at home at night in the darkness with myself, that's when I can't fake anything and that's when all these things come out, and that's when I would [think] "What if no one ever loves me, what if I can never find somebody to love this infected person, what if I die and my parents have to take care of me." All these things that I shouldn't have to think about [...] I have to think about every night[...]

A recurring and unsolicited theme that emerged from these interviews was suicidal ideation. Six of the 14 participants reported having attempted suicide or having frequent thoughts of suicide. All stated that they would not hurt themselves but had thought about it in detail or had failed attempts in the past.

In addition to the high prevalence of depression, all participants indicated past or current substance use. Substances used included alcohol, crystal meth, crack, cocaine, ecstasy, heroin, marijuana, and methadone. All participants mentioned substance use as a direct reason for their inability to sleep or erratic sleep patterns, although marijuana was considered a method to assist in initiating sleep and was used successfully by several participants. Several individuals discussed the impact of these substances in terms of changing one's sleep patterns, experiencing vivid and intense dreams, having "fake sleep" (referring to the participant constantly feeling somnolent with heroin use but being unable to attain deep sleep), not feeling rested, and staying up all night or for several days and then sleeping for numerous days. A 55-year-old Black female on ARVs explained how the need to attend to her heroin addiction did not permit her time to sleep:

[Heroin] affected [my sleep] a lot because I couldn't sleep. I didn't have time to sleep. I had to think about how I'm getting the next one [...] especially when you're a heroin addict and you don't have the money, you don't have time to sleep, you have no time to sleep. You have no time for anything except planning for how you're going to get that next drug. That's it! 
A 35-year-old White male on ARVs was clear about the impact of substance use on his sleep quality: "The crystal meth use where I would be up for three or four days [...] and then crash, that definitely messed up my sleep schedule [...] I don't like to sleep as much anymore."

Eight of the participants stated that they frequently had nightmares. They stated that, at times, the anxiety from the possibility of having nightmares would prevent them from falling asleep, or the fear that they had experienced during a nightmare would frequently wake them up. A 45-yearold Black male on ARVs referred to his dreams as "reality nightmares" because "even though you're asleep they are so real, you actually wake up tired from it."

One $[$ dream] is from childhood [...] the giant Muppets are chasing me down this long hall and I can never get away from them and it's the most terrifying thing in the world $[\ldots]$ And then there's several scenarios that when I was with my partner that beat me [...] and it feels like when I wake up that it just happened, especially the beating dreams and the chasing dreams. It feels like I'm actually running from the people or they're trying to hurt me. [35-year-old White male on ARVs]

A 31-year-old Latino male on ARVs associated his nightmares with the HIV virus, stating: "It's a parasite invading my body. It can't be good. My mind has got to think about it. Subconsciously it's gotta wonder about it. The thing is attacking me and attacking my immune system."

A well-known and commonly reported adverse effect of efavirenz is vivid dreams. Two participants were taking an efavirenz-based ARV regimen at the time of the interview, and another participant had previously taken an efavirenzbased ARV regimen. All three recalled vivid dreams of using illicit substances, feeling the guilt associated with relapsing, and contemplating actually relapsing.

I can visualize the pipe, smoking it, the crazy up feeling you get, and then, I'll wake up and I'll be, well, I could get on the Internet [to obtain illicit drugs]. I could find somebody [a dealer] [...] I don't have any of those phone numbers anymore, but I've learned, living in this city, that there are ways. [35-year-old White male on ARVs]

A 41-year-old Black male on ARVs detailed similar experiences:

There were dreams [...] where I had used and thought that I had relapsed in real life, and [I] went through all those emotions, and I woke up just [...] shaking or like, oh my
God, it's all over, I relapsed. [I could see] everything [...] up to the point of going to get it [...] and putting the crack on the pipe and smoking it, and all the feelings [...] the anticipation, the fear, the anxiety, the fact that I was actually doing this and it felt so real [...] I was like, oh my God! What is my counselor going to say? What is my case worker going to say? They're going to [say] [...] you can't do this, what are you doing? [...] and I'm saying, I can't do this, but I went ahead and did it and then I was devastated [...] It's like, it's all bad, and then it's like but, you did it so you might as well go on and keep doing it.

Disturbing nightmares were also discussed in connection with another recurring and unsolicited mental health theme, the issue of coping with childhood abuse or rape. Six individuals recounted childhood abuse from family members or friends and the impact of these incidents on their current lives (such as the repercussions of running away from home at a young age or engaging in sex work).

I had a recurring abuse nightmare with my uncle. To the point to where I literally would like die; I [would] have to keep my nails cut because I literally gashed into my leg. [45-year-old Black male on ARVs]

\section{Sleep and structural factors}

All participants reported having been homeless, and two were homeless at the time of the interview. These two participants stated that they took very short naps at night and tried to be alert for security reasons. They stated that they woke up frequently due to fear of being assaulted while asleep or robbed for their pocket money. In the words of a 55-yearold Black female not on ARVs: "When you sleep outside, you don't sleep like a normal person sleeps. You have to be careful with yourself."

A 49-year-old American-Indian male on ARVs expressed similar safety concerns: "[They can] beat me to death with a crowbar while I sleep. Hit me in the head [...] or throw gas on me, light me on fire because I'm homeless [...] It's easy to walk up on somebody when they're sleeping and just kill them and they can walk off and get away with it."

One 55-year-old Black female on ARVs who had been homeless 2 years prior to the interview stated that she was still unable to sleep well despite living in a comfortable and safe environment because of the changes in her sleep pattern while homeless: "That's probably why I wake up like I do now. I was so busy having to be on watch [...] You never know what's going to happen, so you definitely can't sleep good there." 
Likewise, two individuals who lived in single occupancy rooms also commented on their inability to sleep due to the intolerable noise level of other occupants and their mistrust of the security of their rooms. One 42-year-old Black male on ARVs stated:

I don't feel comfortable there. I can't sleep for two or three days, I'm going through a lot of mental stress in that building [...] I lock the door and I'd be tired, I still don't sleep well, because $[\ldots]$ people are cruising around my door $[. .$. But, when I do sleep well, I sleep well because I am tired to where it doesn't bother me.

The other, a 41-year-old Black male on ARVs, voiced similar worries:

$[\mathrm{T}]$ hose are the people that I'm concerned about because they know $[\ldots]$ that they could get in [to my room] [...] because this one guy told me once "this dude [...] wouldn't give me something and so, he didn't know that I came in his room and sat by his bedside, and I could've done anything I wanted to do $[\ldots]$ " So, it $[\ldots]$ planted in the back of my mind that, somebody could do that at my house [...] Some nights I would lock the window if I was really tripping that hard[...]

Finally, eleven of the participants had a history of incarceration. Of these, three distinctly described their experiences in prison as contributing to their sleep difficulties. These experiences included the requirement to wake up extremely early to eat breakfast according to the prison schedule, receive medications by standing in "pill-call," and arrive at various appointments.

They had a whole different schedule in jail [...] So, dealing with those stresses it was like that set a pattern for me for those six and a half months [...] getting up at 3:30 in the morning for breakfast, or if you had an appointment outside they would wake you up that early, and then they had pillcall like an hour later [...] Maybe that's still sticking with me. [41-year-old Black male on ARVs]

In addition, participants described the sleep-disturbing effects of the trauma associated with witnessing and experiencing violence in correctional facilities. A 49-year-old American-Indian male on ARVs acknowledged using diazepam, clonazepam, mirtazepine, and quetiapine to reduce his incarceration-related anxiety and to help him sleep.

I get anxiety attacks, panic attacks [...] Killing, I've seen a lot of killing in prison [...] But, when you're in there you learn how to survive too. You learn the ropes. Step over bod- ies, you leave them there, you don't say nothing to nobody, you just step over them, go do what you're supposed to do.

\section{Sleep and physical conditions}

Among the participants, ten stated that physical issues related to ARV medication adverse effects, HIV infection, or sleeping conditions were resulting in poor QOS. These physical issues consisted of flatulence, diarrhea, cramps and spasms in extremities, back and neck pain, toothache, headache, pain associated with prior trauma, asthma and breathing difficulties, and cancer pain. Most participants noted their need to take substantial quantities of pain medications, including over-thecounter, prescription, and illicit drugs, for pain relief and sleep.

In addition, seven of the participants discussed the issue of excessive sweating while sleeping, waking up from the discomfort, and being unable to fall back asleep. A 31-year-old White male on ARVs described it as such: "Night sweats: wake up because you're so soaking wet from sweat. Then you can't go back to sleep because you're freezing [...] I have night sweats all the time [...] You have to change your clothes."

Individuals discussed using fans at night, placing towels on their bed and pillow as precaution, not sleeping due to the fear of waking up drenched, and not wanting to be intimate due to feeling self-conscious of their excessive sweating.

\footnotetext{
Not too many people are going to sleep through putting themselves in a river of sweat [...] I've been in several relationships in my life $[\ldots]$ but even the ones that weren't partners and we laid together in the bed, that was an issue. Because some people [...] wanted to cuddle, they wanted to be close, and I'm like, no, because I'm going to sweat or, you know, it gets too hot[...]. [41-year-old Black male on ARVs]
}

\section{Discussion}

These qualitative interviews suggest a complex and multidimensional impact of mental health issues (such as depression, anxiety, suicidal ideation, substance use, childhood abuse, and dreams), structural factors (such as living situations and incarceration), and physical conditions (such as pain and night sweats) on the sleep quality of marginalized HIV-infected individuals. In general, participants did not associate changes in their QOS with initiation of ARV medications and had not related any deterioration in their sleep quality to seroconversion; however, there were reports of physical issues due to ARV adverse effects and HIV infection and psychological issues due to fear and anxiety surrounding their HIV status. Those reporting nonadher- 
ence due to falling asleep before dosing time or feeling too tired to take medications also reported having lower ARV adherence.

Despite the significant impact of sleep quality on their lives, most participants had not discussed this issue with their providers. This omission may have been due to the overwhelming number of psychosocial issues that may have taken precedence (ie, passive reluctance to discuss QOS due to competing priorities) or a quick-to-prescribe view on the medical establishment, which would result in the need for the participant to take more medications, potentially experience more adverse effects, or be less alert if living on the street. Correspondingly, it was evident that many participants were uninformed of effective approaches to enhance sleep given their self-reported methods, such as alcohol, cigarettes, long-term use of antihistamines, watching television, and substance use, which may be ineffective or can exacerbate insomnia and result in further sleep disruption. A few noted improvements in QOS with exercise and exertion, reading, and creative writing. Of those taking prescription medications for sleep, depression, and anxiety, few indicated major improvements in their QOS.

All participants reported feeling depressed or being diagnosed with depression. The relationship between depression and QOS is closely intertwined, as those with depressive disorders often report high rates of poor QOS, ${ }^{37,38}$ and vice versa. ${ }^{39-42}$ Due to this close correlation, there has been much research and debate regarding the chronology between sleep disorders and depression. ${ }^{43-46}$ Poor QOS in depressed individuals can interfere with daytime functioning and result in a reduction in adherence to treatment. ${ }^{16}$ In a study of the relationship between sleep disturbance, depressive symptoms, and ARV adherence in HIV-infected women, sleep quality was negatively associated with adherence in women who were also depressed, suggesting that depression is a possible mediator in the relationship between QOS and adherence. ${ }^{17}$

A frequently recurring and unsolicited theme that was recounted by nearly half of our sample was suicidal ideation, which may have been related to depression and therefore associated with poor QOS. ${ }^{12}$ In the Swiss National Cohort, Keiser and colleagues demonstrated a reduction in suicides when comparing the period prior to ARV therapy to the late ARV therapy era ${ }^{47}$ however, the suicide rates remain more than three times higher in the HIV-infected population compared to the general public.

Few individuals in our study related their sleep difficulties to adverse effects from their ARV medication. A clear association between the use ARVs and changes in QOS has not been demonstrated. ${ }^{5,12,13,48,49}$ Efavirenz, an HIV nonnucleoside reverse transcriptase inhibitor, has been associated with central nervous system side effects. The likelihood of this adverse effect has been reported to be as high as $22 \%-24 \%,{ }^{50,51}$ and vivid dreams, difficulties falling asleep, and night awakenings are frequently reported..$^{14,15,52}$ In two studies, ${ }^{12,49}$ despite the fact that the use of efavirenz resulted in changes in stages or report of vivid dreams, participants remained satisfied with their QOS or did not report changes in sleep patterns. However, two of our study participants who were former drug users and had taken efavirenz discussed increased awakening due to vivid dreams and experiencing distress due to dreams regarding drug relapse.

The relationship between QOS and childhood abuse, posttraumatic stress from suffering or witnessing violence, depression, HIV, homelessness, incarceration, substance use, and physical issues have been described in prior research: ${ }^{11,12,53-60}$ however, these issues have often not been studied in conjunction with each other and have infrequently been examined in a marginalized HIV-infected population. Results of our qualitative research extend findings from these studies, examine various self-reported correlates of sleep quality simultaneously, describe the association between QOS and ARV adherence, and reveal the lack of disclosure but willingness to speak about sleep problems to health care providers and the level of information regarding sleep-enhancing methods in a marginalized HIV-infected population.

A limitation of our exploratory study is that the purposeful sampling of our patient population was restricted to individuals with poor QOS in the waitlist control arm of the PATH Project; therefore, our results may not be generalizable to other populations. However, the intention of this study was to generate a preliminary account of QOS, ARV adherence, and other correlates in HIV-infected individuals. The assessment of the generalizability of our findings and their utilization in quantitative studies will be the focus of our future research. Additionally, we relied on a self-reported medication adherence measure which tends to be an overestimate of true adherence; therefore, future research should estimate adherence using multiple methods, such as pharmacy refill records or electronic drug monitoring.

From a research standpoint, forthcoming QOS studies in HIV-infected individuals should include questions regarding ARV adherence, mental health issues (such as depression, anxiety, suicidal ideation, substance use, childhood abuse, trauma, and dreams), structural factors (such as living situations and incarceration), physical conditions (such as pain 
and night sweats), and disclosure of sleep difficulties to health care providers. Additionally, future research should examine the efficacy of patient-tailored interventions, using methods such as education and counseling on sleep hygiene, cognitive behavioral therapy, and pharmacologic approaches for the treatment of poor QOS. Studies should determine changes in sleep quality in individuals newly diagnosed with HIV and if the recognition and timely treatment of sleep difficulties in those who report ARV nonadherence due to sleep problems can result in improved adherence. Finally, due to insufficient research regarding QOS in HIV-infected individuals, we relied on several dated references. Due to the prevalence of sleep difficulties among people living with HIV, we believe that emphasis should be placed on the execution of welldesigned research in this area.

Findings from these interviews suggest that QOS may be among various factors that can influence ARV adherence. Despite the fact that few participants had mentioned this issue to their provider, they were willing to openly discuss their sleep difficulties, their fears and anxieties surrounding sleep, and other sensitive topics such as suicidal ideation, childhood abuse, and substance use. Therefore, from a clinical perspective, inquiries into a patient's QOS can serve as a gateway to discussing these sensitive topics, because QOS questions are less emotionally charged, more socially acceptable, and can be conducted using a few questions. QOS can be assessed by asking questions about the patient's bedtime, the time they raise, number of times they wake up in the middle of the night and the approximate duration of these awakenings, difficulty falling asleep or staying asleep, night terrors or night sweats, feeling fatigued in the morning, and struggling to stay awake during the day. Findings regarding poor sleep quality should prompt an assessment of ARV adherence and depression, potentially an evaluation of suicidal ideation and substance use, sleep hygiene counseling, and possible referral to sleep medicine specialists.

\section{Acknowledgments}

We thank Eunice Stephens, Justin Bailey, Rebecca Sedillo, and Samantha Dilworth for their support throughout this project.

\section{Disclosure}

This project was supported by NIH/NIMH grant numbers F32MH086323, K23MH097649, K24MH087220, and R01MH0790700 and NIH/NCRR UCSF-CTSI grant number UL1RR024131. Its contents are solely the responsibility of the authors and do not necessarily represent the official views of the NIH. The authors report no other conflicts of interest in this work.

\section{References}

1. Norman SE, Resnick L, Cohn MA, Duara R, Herbst J, Berger JR. Sleep disturbances in HIV-seropositive patients. JAMA. 1988;260(7): 922.

2. Darko DF, McCutchan JA, Kripke DF, Gillin JC, Golshan S. Fatigue, sleep disturbance, disability, and indices of progression of HIV infection. Am J Psychiatry. 1992;149(4):514-520.

3. Norman SE, Chediak AD, Freeman C, et al. Sleep disturbances in men with asymptomatic human immunodeficiency (HIV) infection. Sleep. 1992;15(2):150-155.

4. Wiegand M, Möller AA, Schreiber W, Krieg JC, Holsboer F. Alterations of nocturnal sleep in patients with HIV infection. Acta Neurol Scand. 1991;83(2):141-142.

5. Rubinstein ML, Selwyn PA. High prevalence of insomnia in an outpatient population with HIV infection. J Acquir Immune Defic Syndr Hum Retrovirol. 1998;19(3):260-265.

6. Ford DE, Kamerow DB. Epidemiologic study of sleep disturbances and psychiatric disorders. An opportunity for prevention? JAMA. 1989;262(11):1479-1484.

7. Quera-Salva MA, Orluc A, Goldenberg F, Guilleminault C. Insomnia and use of hypnotics: study of a French population. Sleep. 1991; 14(5):386-391.

8. Motivala SJ, Sarfatti A, Olmos L, Irwin MR. Inflammatory markers and sleep disturbance in major depression. Psychosom Med. 2005;67(2): 187-194.

9. Mills PJ, Dimsdale JE. Sleep apnea: a model for studying cytokines, sleep, and sleep disruption. Brain Behav Immun. 2004;18(4):298-303.

10. Krueger JM, Obál FJ, Fang J, Kubota T, Taishi P. The role of cytokines in physiological sleep regulation. Ann N Y Acad Sci. 2001;933: 211-221.

11. Benca RM. Sleep in psychiatric disorders. Neurol Clin. 1996;14(4): 739-764.

12. Saberi P, Neilands TB, Johnson MO. Quality of sleep: associations with antiretroviral nonadherence. AIDS Patient Care STDS. 2011;25(9): 517-524.

13. Nokes KM, Kendrew J. Correlates of sleep quality in persons with HIV disease. J Assoc Nurses AIDS Care. 2001;12(1):17-22.

14. Núñez M, González de Requena D, Gallego L, Jiménez-Nácher I, González-Lahoz J, Soriano V. Higher efavirenz plasma levels correlate with development of insomnia. J Acquir Immune Defic Syndr. 2001; 28(4):399-400.

15. Gallego L, Barreiro P, del Río R, et al. Analyzing sleep abnormalities in HIV-infected patients treated with efavirenz. Clin Infect Dis. 2004;38(3):430-432.

16. Thase ME. Antidepressant treatment of the depressed patient with insomnia. J Clin Psychiatry. 1999;60(Suppl 17):28-31; discussion 46-48.

17. Phillips KD, Moneyham L, Murdaugh C, et al. Sleep disturbance and depression as barriers to adherence. Clin Nurs Res. 2005;14(3): 273-293.

18. Paterson DL, Swindells S, Mohr J, et al. Adherence to protease inhibitor therapy and outcomes in patients with HIV infection. Ann Intern Med. 2000;133(1):21-30.

19. Gifford AL, Bormann JE, Shively MJ, Wright BC, Richman DD, Bozzette SA. Predictors of self-reported adherence and plasma HIV concentrations in patients on multidrug antiretroviral regimens. JAcquir Immune Defic Syndr. 2000;23(5):386-395.

20. Little SJ, Holte S, Routy JP, et al. Antiretroviral-drug resistance among patients recently infected with HIV. $N$ Engl J Med. 2002;347(6): 385-394.

21. Grant RM, Hecht FM, Warmerdam M, et al. Time trends in primary HIV-1 drug resistance among recently infected persons. JAMA. 2002; 288(2):181-188. 
22. Simon V, Vanderhoeven J, Hurley A, et al. Evolving patterns of HIV-1 resistance to antiretroviral agents in newly infected individuals. AIDS. 2002;16(11):1511-1519.

23. Blower SM, Aschenbach AN, Gershengorn HB, Kahn JO. Predicting the unpredictable: transmission of drug-resistant HIV. Nat Med. 2001;7(9): 1016-1020.

24. Roth T, Ancoli-Israel S. Daytime consequences and correlates of insomnia in the United States: results of the 1991 National Sleep Foundation Survey. II. Sleep. 1999;22 Suppl 2:S354-S358.

25. Ustinov Y, Lichstein KL, Wal GS, Taylor DJ, Riedel BW, Bush AJ. Association between report of insomnia and daytime functioning. Sleep Med. 2010;11(1):65-68.

26. Breslau N, Roth T, Rosenthal L, Andreski P. Sleep disturbance and psychiatric disorders: a longitudinal epidemiological study of young adults. Biol Psychiatry. 1996;39(6):411-418.

27. Hatoum HT, Kong SX, Kania CM, Wong JM, Mendelson WB. Insomnia, health-related quality of life and healthcare resource consumption. A study of managed-care organisation enrollees. Pharmacoeconomics. 1998;14(6):629-637.

28. Entzian P, Linnemann K, Schlaak M, Zabel P. Obstructive sleep apnea syndrome and circadian rhythms of hormones and cytokines. Am J Respir Crit Care Med. 1996;153(3):1080-1086.

29. Vgontzas AN, Papanicolaou DA, Bixler EO, et al. Circadian interleukin-6 secretion and quantity and depth of sleep. J Clin Endocrinol Metab. 1999;84(8):2603-2607.

30. Irwin MR, Wang M, Campomayor CO, Collado-Hidalgo A, Cole S. Sleep deprivation and activation of morning levels of cellular and genomic markers of inflammation. Arch Intern Med. 2006;166(16): 1756-1762.

31. Akerstedt T, Nilsson PM. Sleep as restitution: an introduction. J Intern Med. 2003;254(1):6-12.

32. Shamsuzzaman AS, Gersh BJ, Somers VK. Obstructive sleep apnea: implications for cardiac and vascular disease. JAMA. 2003;290(14): 1906-1914.

33. Minoguchi K, Yokoe T, Tazaki T, et al. Increased carotid intima-media thickness and serum inflammatory markers in obstructive sleep apnea. Am J Respir Crit Care Med. 2005;172(5):625-630.

34. Buysse DJ, Reynolds CF 3rd, Monk TH, Berman SR, Kupfer DJ. The Pittsburgh Sleep Quality Index: a new instrument for psychiatric practice and research. Psychiatry Res. 1989;28(2):193-213.

35. Thomas DR. A general inductive approach for analyzing qualitative evaluation data. American Journal of Evaluation. 2006;27(2):237-246.

36. Bradley EH, Curry LA, Devers KJ. Qualitative data analysis for health services research: developing taxonomy, themes, and theory. Health Serv Res. 2007;42(4):1758-1772.

37. Jansson M, Linton SJ. The role of anxiety and depression in the development of insomnia: cross-sectional and prospective analyses. Psychology and Health. 2006;21(3):383-397.

38. Morphy H, Dunn KM, Lewis M, Boardman HF, Croft PR. Epidemiology of insomnia: a longitudinal study in a UK population. Sleep. 2007; 30(3):274-280.

39. Morgan K, Healey DW, Healey PJ. Factors influencing persistent subjective insomnia in old age: a follow-up study of good and poor sleepers aged 65 to 74. Age Ageing. 1989;18(2):117-122.

40. Charon F, Dramaix M, Mendlewicz J. Epidemiological survey of insomniac subjects in a sample of 1,761 outpatients. Neuropsychobiology. 1989;21(3):109-110.

Patient Preference and Adherence

\section{Publish your work in this journal}

Patient Preference and Adherence is an international, peer-reviewed, open access journal focusing on the growing importance of patient preference and adherence throughout the therapeutic continuum. Patient satisfaction, acceptability, quality of life, compliance, persistence and their role in developing new therapeutic modalities and compounds to
41. Riemann D, Voderholzer U. Primary insomnia: a risk factor to develop depression? J Affect Disord. 2003;76(1-3):255-259.

42. Taylor DJ, Lichstein KL, Durrence HH. Insomnia as a health risk factor. Behav Sleep Med. 2003;1(4):227-247.

43. Soldatos CR. Insomnia in relation to depression and anxiety: epidemiologic considerations. J Psychosom Res. 1994;38 Suppl 1:3-8.

44. Riemann D, Berger M, Voderholzer U. Sleep and depression - results from psychobiological studies: an overview. Biol Psychol. 2001; 57(1-3):67-103.

45. Jansson-Fröjmark M, Lindblom K. A bidirectional relationship between anxiety and depression, and insomnia? A prospective study in the general population. J Psychosom Res. 2008;64(4):443-449.

46. Jansen PW, Saridjan NS, Hofman A, Jaddoe VW, Verhulst FC, Tiemeier H. Does disturbed sleeping precede symptoms of anxiety or depression in toddlers? The generation R study. Psychosom Med. 2011;73(3):242-249.

47. Keiser O, Spoerri A, Brinkhof MW, et al; for Swiss HIV Cohort Study; Swiss National Cohort. Suicide in HIV-infected individuals and the general population in Switzerland, 1988-2008. Am J Psychiatry. 2010;167(2):143-150.

48. Wheatley D, Smith K. Clinical sleep patterns in human immune virus infection. Hum Psychopharmacol. 1994;9(2):111-115.

49. Moyle G, Fletcher C, Brown H, Mandalia S, Gazzard B. Changes in sleep quality and brain wave patterns following initiation of an efavirenz-containing triple antiretroviral regimen. HIV Med. 2006;7(4): 243-247.

50. Lochet P, Peyrière H, Lotthé A, Mauboussin JM, Delmas B, Reynes J. Long-term assessment of neuropsychiatric adverse reactions associated with efavirenz. HIV Med. 2003;4(1):62-66.

51. Goldenberg D, Boyle B. Psychiatric safety of efavirenz. Proceedings of the 13th International AIDS Conference; 2000 Jul 9-14; Durban, South Africa.

52. Fumaz CR, Tuldrà A, Ferrer MJ, et al. Quality of life, emotional status, and adherence of HIV-1-infected patients treated with efavirenz versus protease inhibitor-containing regimens. J Acquir Immune Defic Syndr. 2002;29(3):244-253.

53. Davis JE, Shuler PA. A biobehavioral framework for examining altered sleep-wake patterns in homeless women. Issues Ment Health Nurs. 2000;21(2):171-183.

54. Greenfield EA, Lee C, Friedman EL, Springer KW. Childhood abuse as a risk factor for sleep problems in adulthood: evidence from a US national study. Ann Behav Med. 2011;42(2):245-256.

55. Humphreys JC, Lee KA, Neylan TC, Marmar CR. Sleep patterns of sheltered battered women. Image J Nurs Sch. 1999;31(2):139-143.

56. Lee KA, Gay C, Portillo CJ, et al. Types of sleep problems in adults living with HIV/AIDS. J Clin Sleep Med. 2012;8(1):67-75.

57. Peterson MJ, Benca RM. Sleep in mood disorders. Psychiatr Clin North Am. 2006;29(4):1009-1032; abstract ix.

58. Schierenbeck T, Riemann D, Berger M, Hornyak M. Effect of illicit recreational drugs upon sleep: cocaine, ecstasy and marijuana. Sleep Med Rev. 2008;12(5):381-389.

59. Teplin D, Raz B, Daiter J, Varenbut M, Tyrrell M. Screening for substance use patterns among patients referred for a variety of sleep complaints. Am J Drug Alcohol Abuse. 2006;32(1):111-120.

60. Vosvick M, Gore-Felton C, Ashton E, et al. Sleep disturbances among HIV-positive adults: the role of pain, stress, and social support. $J$ Psychosom Res. 2004;57(5):459-463.

\section{Dovepress}

optimize clinical outcomes for existing disease states are major areas of interest. This journal has been accepted for indexing on PubMed Central. The manuscript management system is completely online and includes a very quick and fair peer-review system. Visit http://www.dovepress.com/ testimonials.php to read real quotes from published authors. 